\title{
FOURIER BASED REGISTRATION OF DIFFERENTIALLY SCALED IMAGES
}

\author{
Ruben Gonzalez
}

Griffith University, Gold Coast Campus, QLD, Australia,

\begin{abstract}
This paper presents a novel Fourier based approach for registration of images that have been translated, rotated, scaled and stretched. Fourier based techniques are computationally efficient and provide noise resilience but beyond similarity transformed images they do not extend readily to more general affine deformations. The proposed method relies on applying a Hough circle transform to a generated log-polar correlation surface in conjunction with various techniques for noise rejection. Applicable theory, methodology and preliminary results are presented.
\end{abstract}

Index Terms - Affine, Fourier, Registration, Image

\section{INTRODUCTION}

For images that have undergone similarity transformation, Fourier based image registration techniques can provide a robust and efficient solution. A typical approach using Phase Correlation in combination with a log-polar transform [1] can recover a wide range of global rotation, scale and translation parameters. The global nature of Fourier techniques renders them unsuitable for images exhibiting elastic deformation. Yet between similarity transforms and elastic deformation is a large class of more general affine transformations. One example of this type of transform is differential (or anisotropic) scaling. To date there appears little published work in the application of Fourier based image registration techniques to this class of transforms.

Previous work in the use of Fourier methods for estimating affine transformation parameters has mostly relied on directly analysing the Fourier transforms of the candidate images in the context of estimating shape from texture. These have analyzed the second order moments, or first order approximations of the differences of Fourier transforms of the images [2,3]. Other work avoids the use of frequency domain techniques all together as being of unworkable in practice [4]. A recent approach estimates the affine transformation parameters from radial projections of the squared Fourier magnitudes of the images [5].

This paper approaches the task of affine invariant image registration as an extension of the common rotation and scale invariant registration. Section two presents the theoretical concepts; section three discusses methodology, with experimental results presented in section four.

\section{THEORY}

Let $f_{1}(x, y)$ and $f_{2}(x, y)$ be two images where $[x, y] \in \mathfrak{R}^{2}$ and let $f_{2}$ be a translated, rotated and differentially scaled of $f_{1}$ by rotation angle $\theta$, translation $\left(\mathrm{x}_{0}, \mathrm{y}_{0}\right)$ and scale factors $\mathrm{s}_{\mathrm{x}}, \mathrm{s}_{\mathrm{y}}$, such that $f_{1}$ is related to $f_{2}$ by the following transformation:

$$
f_{2}(x, y)=f_{1}(j, k)
$$

Where: $\quad \begin{aligned} & j=s_{x} \cdot x \cos \theta-s_{y} \cdot y \sin \theta-x_{0} \\ & k=s_{x} \cdot x \sin \theta+s_{y} \cdot y \cos \theta-y_{0}\end{aligned}$

Taking the Fourier transform of both sides of (1) and using the shifting, scaling and rotation theorems gives:

$$
\begin{array}{r}
F_{2}(u, v)=\frac{1}{\left|s_{x} \cdot s_{y}\right|} e^{-j\left(u x_{0}+v y_{0}\right)} \times F_{1}\left(u / s_{x} \cos \theta-v / s_{x} \sin \theta,\right. \\
\left.u / s_{y} \cos \theta+v / s_{y} \sin \theta\right)
\end{array}
$$

Taking the magnitude of (3) such that $G_{\mathrm{i}}=\left|F_{\mathrm{i}}\right|$ gives:

$$
\begin{array}{r}
G_{2}(u, v)=\frac{1}{\left|s_{x} \cdot s_{y}\right|} G_{1}\left(u / s_{x} \cos \theta-v / s_{x} \sin \theta,\right. \\
\left.u / s_{y} \cos \theta+v / s_{y} \sin \theta\right)
\end{array}
$$

Denoting the Cartesian coordinates $(u, v)$ on the RHS of (4) in terms of the polar coordinates $(r, \phi)$ by substituting for $u=r \cos \phi$ and $v=r \sin \phi$, gives:

$$
\begin{array}{r}
G_{2}(u, v)=\frac{1}{\left|s_{x} \cdot s_{y}\right|} G_{1}\left(r / s_{x} \cos \theta \cos \phi-r / s_{x} \sin \theta \sin \phi,\right. \\
\left.r / s_{y} \sin \theta \cos \phi+r / s_{y} \cos \theta \sin \phi\right)
\end{array}
$$

Applying the product to sum trigonometric identities results in the following relationship:

$$
G_{2}(u, v)=\frac{1}{\left|s_{x} \cdot s_{y}\right|} G_{1}\left(r / s_{x} \cos (\phi+\theta), r / s_{y} \sin (\phi+\theta)\right.
$$

Mapping expression (6) into polar coordinates $(r, \phi)$ where $\boldsymbol{r}=\sqrt{\mathrm{u}^{2}+\mathrm{v}^{2}}$, and $\phi=\arctan (v / u)$ and ignoring the scaling factor results in:

$$
G_{2}(r, \phi)=G_{1}\left(\frac{r}{s_{y}} \sqrt{\beta}, \phi+\tau\right)
$$


where $\quad \tau=\arctan \left(\frac{s_{x}}{s_{y}} \tan (\phi+\theta)\right)-\phi$

and

$$
\beta=\frac{s_{y}^{2}}{s_{x}^{2}} \cos ^{2}(\phi+\theta)+\sin ^{2}(\phi+\theta)
$$

Which can be reformulated as:

$$
\beta=z-m \sin (\sigma)
$$

where: $\quad z=\frac{s_{y}^{2}}{s_{x}^{2}}+\frac{1}{2} \quad m=\frac{1}{2}\left(\frac{s_{y}^{2}}{s_{x}^{2}}-1\right) \quad \sigma=2(\theta+\phi)+\frac{\pi}{2}$

Taking the logarithm of the first variable in the expression in (7) converts the multiplications into additions to give:

$$
G_{2}(\ln [r], \phi)=G_{1}\left(\ln [r]-\ln \left[s_{y}\right]+\frac{1}{2} \ln [\beta], \phi+\tau\right)
$$

Let $\rho=\ln [r], k=-\ln \left[s_{y}\right], b=1 / 2 \ln [\beta]$ and relabeling these relationships as $h_{2}(\omega, \phi)=G_{2}(\rho, \phi)$ and $h_{1}(\omega, \phi)=G_{1}(\rho, \phi)$ results in the Fourier transform of $\mathrm{H}_{2}$ being given by:

$$
\begin{aligned}
& \mathcal{F}\left\{h_{2}(\omega, \phi)\right\}= H_{2}(\varepsilon, \alpha) \\
&=\frac{1}{\sqrt{2 \pi}} \iint G_{1}(\omega, \phi) \cdot e^{-i 2 \pi(\varepsilon(\omega+k)+\alpha(\theta+\tau))} \\
& \times e^{-i 2 \pi \cdot \varepsilon \cdot b} d \omega d \phi
\end{aligned}
$$

Which can be simplified to:

$$
H_{2}(\varepsilon, \alpha)=H_{1}(\varepsilon, \alpha) \cdot e^{-i(\varepsilon k+\alpha \tau)} \int e^{-i 2 \pi \cdot \varepsilon \cdot b} d \phi
$$

Now the remaining integral in (14) can be expressed in the general form of an $\mathrm{n}^{\text {th }}$ order Bessel function of the first kind, denoted as $J_{n}(m)$. Accordingly we rewrite (13) as follows:

$$
H_{2}(\varepsilon, \alpha)=H_{1}(\varepsilon, \alpha) \cdot e^{-i(\varepsilon k+\alpha \tau)} \cdot J_{n}(m)
$$

Taking the normalized cross power spectrum of (15) factors out the phase difference since the magnitude of a complex exponential is simply the radius of a unit circle:

$$
\frac{H_{1}(u, v) \cdot H_{2}^{*}(u, v)}{\left|H_{1}(u, v) \cdot H_{2}^{*}(u, v)\right|}=e^{-i(\varepsilon k+\alpha \tau)} \cdot J_{n}(m)
$$

Finally, the Fourier transform of a Bessel function of the first kind is a ring delta function. Convolving it with a Dirac delta at $(k, \tau)$ shifts the ring's origin, so taking the inverse Fourier transform of (17) gives:

$$
\mathcal{F}^{-1}\left\{e^{-i(\varepsilon k+\alpha \tau)} \cdot J_{n}(m)\right\}=\delta(\varepsilon+k, \alpha+\tau) * \operatorname{Ring}(m)
$$

From the result in (17) we can simultaneously obtain the global scale of the transformed image from $k$, its rotation from $\tau$ and the differential scale from $m$ as described in the following section.

\section{METHOD DESCRIPTION}

Obtaining the transformation parameters of an image pair where one has been undergone anisotropic scaling in addition to rotation and translation begins in the same manner as that for recovery of similarity transformations. First a translation invariant representation of the images is obtained by making use of the Fourier shift theorem. The images are then remapped into the log polar domain converting rotation and scaling into shifts. Phase correlation is then applied to find the rotation and isotropic scaling parameters. The conversion of the source image $f(x, y)$ in the Cartesian space into the log-polar domain $g(\rho, \varphi)$ is performed using bilinear interpolation by resampling the magnitude of the $\mathrm{N} x \mathrm{~N}$ FFTs on an N/2 x N/2 log-polar grid using the relationship:

$$
g(\rho, \varphi)=f\left(N / 2+\beta^{y} \cdot \cos (\alpha), N / 2+\beta^{y} \cdot \sin (\alpha)\right)
$$

Where the variables $\boldsymbol{\alpha}$ and $\boldsymbol{\beta}$ are given by:

$$
\alpha=\frac{\pi \mathrm{x}}{\mathrm{N}} \quad \& \quad \beta=e^{\log (N / 2) / N / 2}
$$

The location of the peak on the resulting correlation surface indicates the rotation and scaling relation between the source images. The rotation angle $\theta$ and the scale $S$ are recovered as:

$$
\theta=\frac{\pi \varphi}{N} \quad \& \quad S=N^{1 / N^{\rho}}
$$

The translation can also be recovered using standard phase correlation after application of the inverse transform to compensate for the scale and rotation differences.

Anisotropic scaling can be considered as a distortion of a similarity transform. This distortion disperses the "correlation energy" from any would be peak by a distance related by the amount of distortion. The pattern of this dispersal is to concentrate energy into a ring situated around the location determined by the rotation and isotropic scale of the image. The location and size of this ring can be found via a Hough circle transform (HCT). With increasing distortion, the increasing energy dispersal significantly lowers the signal to noise in proportion to the size of the ring. The intensity of the peaks making up the ring relative to the noise is such that the HCT alone is often unlikely to correctly identify the ring. This can be effectively resolved with a combination of pre and post processing techniques.

First, noise can be excluded from consideration by the HCT through appropriate filtering. As the noise tends to be randomly distributed, local noise averaging can be used to reduce its significance. Each point in the correlation surface is renormalized according to the the mean local noise:

$$
n(x, y)=\frac{1}{4 r^{2}} \sum_{i=-r}^{+r} \sum_{j=-r}^{+r} f(x, y) \quad f(x, y)=\frac{f(x, y)}{n(x, y)}
$$

Another technique that can be applied to improve the reliability of ring detection is the application of a high frequency emphasis filter as reported in [6]. By increasing the signal to noise ratio of the correlation surface these methods improve the success of the circle detection. Postprocessing can also be used to reject HCT misdetections by considering the statics of the points comprising candidate 
ring. The resulting HCT peaks are ranked, a shortlist selected, and the inverse of the coefficient of variation (ICV) of each implied ring $j$ in the list is calculated along its circumference. Using polar coordinates the equation of a circle centred at $\left(\rho_{\mathrm{j}}, \phi_{\mathrm{j}}\right)$ with radius $\mathrm{r}_{\mathrm{j}}$ is given by:

$$
C_{j}(\theta)=\rho_{j} \cos \left(\theta-\phi_{j}\right)+\sqrt{r_{j}^{2}-\rho_{j}^{2} \sin ^{2}\left(\theta-\phi_{j}\right)}
$$

The inverse coefficient of variation is defined, where $n_{k}$ is the number of points in the circumference, as follows:

$$
\begin{aligned}
& \mu_{j}=\frac{1}{n_{j}} \oint_{C_{j}} f(\theta) \cdot d \theta \\
& I C V_{j}=\frac{\mu_{j}}{\sqrt{\frac{1}{n_{j}-1} \oint_{C_{j}}\left(f(\theta)-\mu_{j}\right)^{2} \cdot d \theta}}
\end{aligned}
$$

The ring with the largest resulting inverse coefficient of variation is selected from the shortlist as the most likely one. The radius of the ring gives the amount of anisotropic scaling factor between the two images but not the orientation. The centroid of the ring $(k, \tau) \equiv\left(\rho_{\mathrm{j}}, \phi_{\mathrm{j}}\right)$ gives the rotation and isotropic scale shifted by the radius of the ring. The various steps in this process is depicted in Fig.1

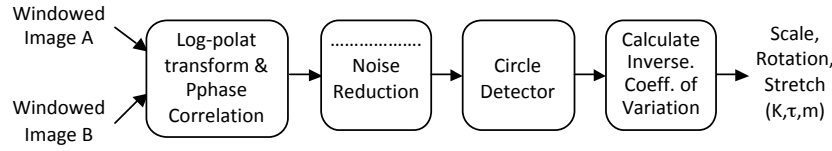

Fig. 1. Calculation of transformation parameters

The amount of anisotropic distortion as a ratio of the isotropic scaling where $m$ is the ring radius and $\mathrm{W}$ is half the number of points in the final Fourier transform, is given by:

$$
\frac{S_{y}}{S_{x}}=\left(W^{1 / W}\right)^{m}
$$

The isotropic scale factor $S$ can then be recovered similarly to the case of similarity transforms given in (19) with $k$ substituted for $\rho$ by first subtracting the ring radius.

$$
S=\left(N^{1 / N}\right)^{(\rho-m)}
$$

For stretch ratios from $1.0 \ldots 2.0$, the rotation angle $\theta$ can be recovered using of the first order approximation of (8) which cancels out $\phi$ and substituting $\tau$ for $\varphi$ in (20):

$$
\theta=\frac{\tau \cdot \pi}{N}
$$

the scaling ratio from (24) and the angular component of the centre of the ring $\tau$, the rotation angle $\theta$ can be recovered from (8) with letting $\phi=0$ as:

$$
\theta=\frac{\pi}{N} \arctan \left(\frac{s_{x}}{s_{y}} \tan (\tau)\right)
$$

After the isotropic and anisotropic scaling and rotation parameters have been recovered, the relevant image is can be inverse transformed and phase correlation applied one more time to recover the translation parameters.

\section{EXPERIMENTAL RESULTS}

Results of the processing steps applied to a pair of images generated by stretching by a factor of 1.4 is shown in Figure 2. The subimage at the topleft shows the original reference image with the transformed replica below it. The top centre subimage is a negative image of the resulting correlation surface that shows a very faint ring in the centre of a magnitude much lower than the noise level. The subimage immediately below it shows the result of applying the noise reduction of (20). To its far right is the result of the circle detection using the HCT and ICV. The location of strongest ring in the centre indicates the transformation consists of simple stretching. The other faint rings show the top five most likely circles from the HCT.

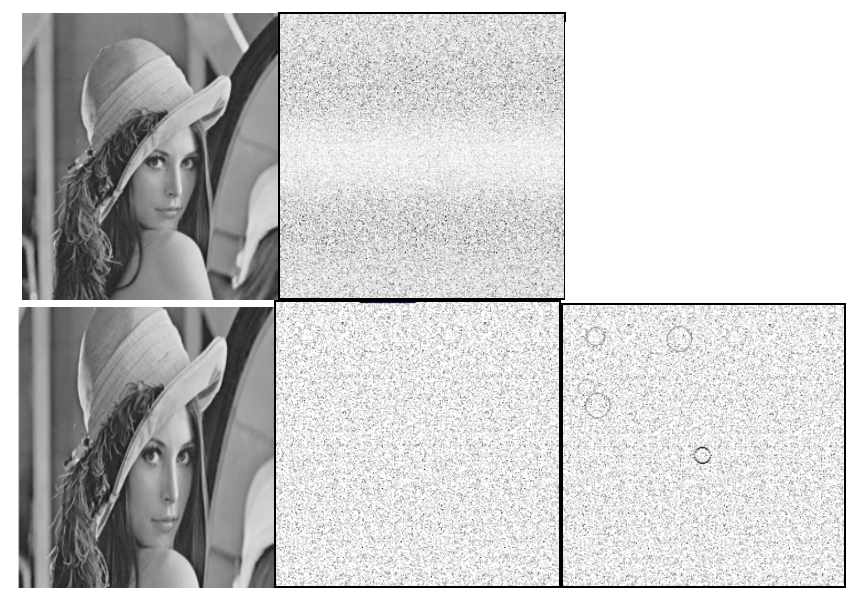

Fig. 2. Vertical stretching by a factor of $1.4 \mathrm{x}$

Figure 3 shows the result of applying isotropic scaling followed by stretching and rotation. The left subimage is the transformed source, the centre image is the input to the circle detection showing a faint ring offset from the centre and the detected ring is shown on the right.

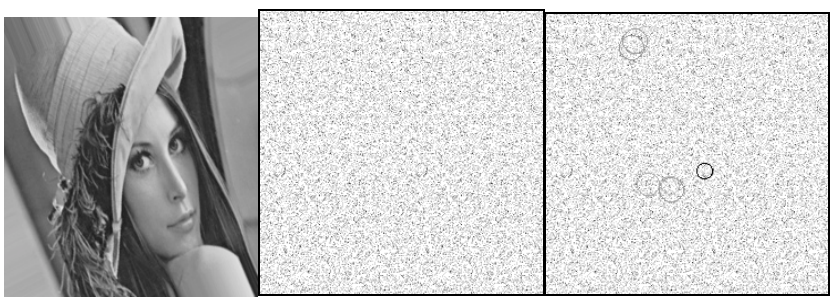

Fig. 3. Affine transformation: scaled by $1.2 \mathrm{x}$ then vertically stretched by a factor of 1.3 followed by a $25^{\circ}$ rotation

Figure 4 shows the result of stretching the source image in an arbirary orietation, without rotation or global scaling. The stretching has no effect on the location or size of the ring which is correctly detected at the centre of the surface. 


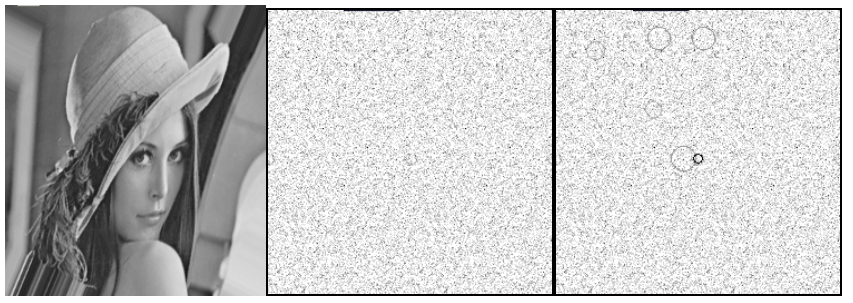

Fig. 4. Arbitrary stretching by $1.25 \mathrm{x}$ at an angle of $-25^{\circ}$

The performance of the proposed scheme was further evaluated using a dataset consisting of geometrically transformed replicas of three different 512x512 images. Each was framed in the centre of a $1024 \times 1024$ border and then replicated a total of 3795 times using a different combination of isotrpic and ansiotropic scaling and rotation factors. This resulted in 11,385 different image pairs where the isotropic scaling ranged from 0.3 to 3.5 in 33 logarithmically uniform steps. The rotation ranged from \pm 180 degrees in 15-degree steps and the images were further stretched by between 0 to $40 \%$ of the isotropic scale.

The experiments focused on the ability of the proposed method to correctly localise the centres of distortion induced the rings for given amounts of scale and rotation at different degrees of image distortion. As a simplification, the ability to reliabily detect the isotropic scale factor was used as the measure of success since the rotation angle does not have a significant influence on the detectability of the rings. For the purpose of these experiments a given scale factor was deemed to be reliably detected if the average error at a given amount of distortion for all rotation angles was below $15 \%$. This error threshold was chosen rather arbitrarily since error distribution tends to be a step function: where the rings are correctly identified the error is typically below $15 \%$, otherwise the average error is above $40 \%$.

The results reveal that the range of isotropic scale factors than can be reliably recovered decreases as stretching increases. This is depicted in Figure 5, which plots the average of the scale factors reliably recovered for different amounts of stretching, across the three test images.

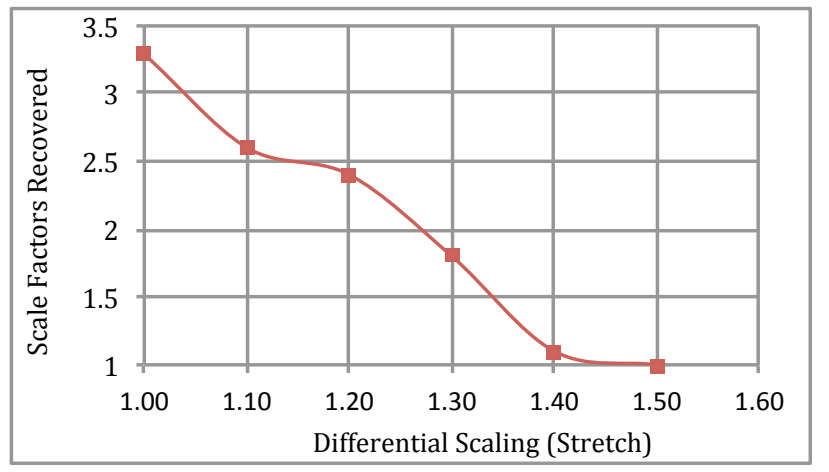

Fig. 5. Reliably recovered transformation parameters as a function of degree of differential scaling.
At moderate levels of isotropic scaling, significant amounts of stretching (around 40\%) can be reliably detected and recovered. Even at scaling by factors greater than $2 \mathrm{x}$, stretching of up to $20 \%$ can be recovered. Accordingly, registration of stretched, rotated, scaled and translated images can be performed with this method.

\section{CONCLUSIONS AND FUTURE WORK}

The theoretical result that differential scaling produces a ring rather than a single peak in log-polar domain Fourier correlation surfaces permits the use of circle detection to recover the transformation parameters. This approach retains the benefits of Fourier based image registration such as constant processing time and robustness to image noise. However in practice the very low signal to noise ratios that result require additional processing to ensure that the rings are reliably detected. The preliminary results presented in this paper show that the approach is viable.

The extension of this approach to detect the orientation of anisotropic scaling and to other types of affine transforms that include shearing components is currently under investigation. As is also, extending the range of scale and distortion factors that may be recovered by improving the reliability of ring detection through additional processing.

\section{REFERENCES}

[1] B.S. Reddy, B.N. Chatterji, "An FFT-based technique for translation, rotation, and scale-invariant image registration", IEEE Trans. on Image Processing, vol.5, no. 8, pp.1266-1271, 1996.

[2] B.J. Super, A.C. Bovik, "Shape from texture using local spectral moments", IEEE Trans. PAMI Apr 1995 Vol.17, No. 4 pp $333-343$

[3] J. Krumm, "Shape from periodic texture using the spectrogram", Computer Vision and Pattern Recognition, 1992. Proceedings CVPR '92, 15-18 Jun 1992, pp 284 - 289

[4] S. Zokai, G. Wolberg, "Image Registration Using Log-Polar Mappings for Recovery of Large-Scale Similarity and Projective Transformations." IEEE Trans. Image Processing, vol.14, no.10, pp.1422-1434, 2005

[5] L. Lucchese, S. Leorin, GM. Cortelazzo, "Estimation of twodimensional affine transformations through polar curve matching and its application to image mosaicking and remotesensing data registration.” IEEE Trans. Image Process. 2006 Oct; Vo.15, No. 10, pp.3008-19.

[6] R. Gonzalez, "Improving Phase Correlation for Image Registration", Proceedings of Image and Vision Computing New Zealand 2011, p.488-493, Nov.29 - Dec.1, http://www.ivs.auckland.ac.nz/ivcnz2011/proceedings.pdf 